\title{
Peristaltic Recovery After Peroral Endoscopic Myotomy for Achalasia: Dream or Reality?
}

TO THE EDITOR: We read with interest the article on esophageal motility patterns after peroral endoscopic myotomy (POEM) in achalasia. ${ }^{1}$ We appreciate the authors' effort to maintain such a large database and a concise presentation. However, we like to draw attention to certain points that we believe might help the readers to understand how much recovery of peristalsis does occur after POEM in these patients more truthfully. The authors have taken the liberty of using the term "recovery of peristalsis," by defining a mere $3-\mathrm{cm}$ long isobaric contour integrity of $20 \mathrm{mmHg}$. The Chicago classification versions 3.0 and $4.0^{2,3}$ define peristalsis as a coordinated contraction with normal distal latency (DL; ie, $\geq 4.5$ seconds) and normal distal contractile integral (ie, $\geq 450 \mathrm{mmHg} \cdot \mathrm{sec} \cdot \mathrm{cm}$ ). Any contraction not fulfilling these criteria, particularly the DL criteria, cannot be labeled as peristalsis; hence, the term "peristaltic recovery" is misleading as even a simultaneous contraction of adequate amplitude may be misunderstood as peristaltic. It would also be overtly reassuring to an interventionist as well as to the patients.

A significant proportion of patients were labeled as esophagogastric junction outflow obstruction (EGJOO) after POEM, especially for achalasia types 2 and 3 . It is also essential to ensure whether a normal peristalsis was seen in these patients or simultaneous contractions fulfilling the arbitrary criteria were labeled as normal peristalsis to diagnose EGJOO. In fact, diagnosis of EGJOO as per standard Chicago classification requires presence of normal peristalsis, which needs to have a DL of $\geq 4.5$ seconds. Similar issue can be raised about the finding of "fragmented peristalsis" after POEM in these patients. The authors have rightly conceded that impedance data showing a post-POEM improvement in esophageal emptying would have been critical in the interpretation of these findings. The standard of care suggests the need for documenting adequate esophageal emptying on timed barium esophagogram as an evidence of adequacy of treatment for achalasia.

Moreover, some studies quoted by the authors are either feline studies $^{4}$ or have used conventional manometry to look for peristalsis, ${ }^{5,6}$ which is not as accurate as high-resolution manometry in analyzing esophageal motility, especially after an intervention.

In conclusion, it is well known that a proportion of patients have altered peristalsis after intervention in achalasia; however, the data provided by the authors are not adequately convincing to suggest that normal esophageal peristalsis returns after POEM for achalasia.

Akshay Kulkarni, Anshuman Elhence, and Uday C Ghoshal* Department of Gastroenterology, Sanjay Gandhi Post Graduate Institute of Medical Sciences, Lucknow, India

1. Vackova Z, Mares J, Krajciova J, et al. Esophageal motility patterns after peroral endoscopic myotomy in patients with achalasia. J Neurogastroenterol Motil 2021;27:205-214.

2. Kahrilas PJ, Bredenoord AJ, Fox M, et al. The Chicago classification of esophageal motility disorders, v3.0. Neurogastroenterol Motil 2015;27:160-174.

3. DeLay K, Yadlapati R, Pandolfino JE. Chicago classification of esophageal motility disorders: past, present, and future. Indian J Gastroenterol 2021;40:120-130.

4. Schneider JH, Peters JH, Kirkman E, Bremner CG, DeMeester TR. Are the motility abnormalities of achalasia reversible? An experimental outflow obstruction in the feline model. Surgery 1999;125:498-503.

5. Zaninotto G, Costantini M, Anselmino M, BoccùC, Ancona E. Onset of oesophageal peristalsis after surgery for idiopathic achalasia. Br J Surg 1995;82:1532-1534.

6. Patti MG, Galvani C, Gorodner MV, Tedesco P. Timing of surgical intervention does not influence return of esophageal peristalsis or outcome for patients with achalasia. Surg Endosc 2005;19:1188-1192.

Financial support: None.

Conflicts of interest: None.

Author contributions: Akshay Kulkarni, Anshuman Elhence, and Uday C Ghoshal conceptualized the letter, wrote and edited it, and approved the final version. 